\title{
First Seroprevalence Study of Coxiella burnetii in Rural Pregnant Women in Contact with Livestock in Khorramabad
}

\author{
Eiman Azizyari Ghobadi ${ }^{1}$, Amin Jaydari (iD ${ }^{1,}{ }^{*}$, Soheila Akbari ${ }^{2}$ and Khaterh Anbari ${ }^{3}$ \\ ${ }^{1}$ Department of Pathobiology, Faculty of Veterinary Medicine, Lorestan University, Khorramabad, Iran \\ ${ }^{2}$ Department of Obstetrics and Gynecology, Lorestan University of Medical Sceinces, Khorramabad, Iran \\ ${ }^{3}$ Department of Community Medicine, Lorestan University of Medical Sciences, Khorramabad, Iran \\ "Corresponding author: Department of Pathobiology, Faculty of Veterinary Medicine, Lorestan University,Khorramabad, Iran. Email: jaydari.a@lu.ac.ir
}

Received 2019 September 05; Revised 2019 October 11; Accepted 2019 October 15.

\begin{abstract}
Background: Coxiella burnetii is the cause of $\mathrm{Q}$ fever. There is no adequate information about $\mathrm{Q}$ fever in rural pregnant women in Iran.

Objectives: The aim of this study was to investigate the seroprevalence of $C$. burnetii infection among rural pregnant women with historical contact with livestock in Khorramabad (western Iran).

Methods: This cross-sectional was conducted from December 2016 to June 2017 on 184 samples collected randomly from rural pregnant women who referred to clinical laboratories and health centers in Khorramabad. The data were examined using indirect ELISA assay kits for the detection of $C$. burnetii phase II human antibodies in their serum samples.

Results: Of 184 serum samples of rural pregnant women, 89 (48.4\%) were positive, 76 (41.3\%) negative, and 19 (10.3\%) suspected. In addition, there was a significant relationship between age, pregnancy, abortion, and the location of the village relative to the city. The results suggested that more positive samples were observed in women that lived in the eastern villages of Khorramabad city; however, the difference was not statistically significant $(\mathrm{P}$ value $=0.535)$.

Conclusions: The present study demonstrated the high seroprevalence of $C$. burnetii in rural pregnant women, which necessitates preventing the unwanted side effects of the disease. Rural pregnant women should pay more attention to the disease and its dangerous effects.
\end{abstract}

Keywords: Coxiella burnetii, Seroprevalence, Rural, Pregnant Women, Khorramabad

\section{Background}

Q fever is a zoonotic disease of humans and animals being reported in various climatic areas. It is considered a public health problem in many countries of the world (1). The cause of the disease is a Gram-negative, obligate intracellular bacterium called Coxiella burnetii (2). This bacterium can be used for a long time outside the host body and transmitted through the respiratory tract to the new host (3). The main source of human infection is domestic and pet animals, including cattle, sheep, goats, dogs, cats, and birds (4). Q fever has acute and chronic forms. The acute form has usually several symptoms like fever, cough, headache, body pain, and fatigue. In the absence of treatment, it can develop a chronic form, with symptoms such as endocarditis, chronic lung infection, and bone infection $(1,5)$.

Coxiella burnetii may result in a number of symptoms in pregnant women such as abortion, intrauterine growth retardation, premature birth, oligoamnios, and spontaneous abortion (6). Serological molecular techniques and culture are used for the diagnosis of C. burnetii. The culture of this bacterium is very dangerous, time-consuming, and expensive. The molecular technique has lower sensitivity than other laboratory methods (7). The best method for diagnosing bacterial serology is the enzyme-linked immunosorbent assay (ELISA) and indirect immunofluorescence antibody (IFA) (8). In this study, the ELISA method was used for the diagnosis of C. burnetii phase II. There is no study on Q fever among rural pregnant women in Iran except for one study done in Ahvaz and Parsabad (9).

\section{Objectives}

Thus, this is the first study that investigated C. burnetii in rural pregnant women with a history of livestock contact in Iran. 


\section{Methods}

In this cross-sectional study, we assessed the seroprevalence rate of Coxiella burnetii in rural pregnant women with historical contact with livestock referring to clinical and health centers in Khorramabad from December 2016 to June 2017. A number of questions were asked about the age of the patient, the patient's residence place, history of contact with livestock, abortion history, gestational age, etc. Samples of blood were randomly collected and centrifuged at $3500 \mathrm{~g}$ for $5 \mathrm{~min}$. Sera samples were transferred on ice to the faculty of veterinary and kept at $-20^{\circ} \mathrm{C}$ until analysis. The samples were tested for the presence of IgG antibodies against $C$. burnetii phase II using an indirect ELISA test commercial kit (Vircell, SL, Granada, Spain, no. G1001). Based on the manufacturer's kit, the positive control OD was $>0.9$, negative control OD $<0.5$, and cutoff control OD $>0.55$ and $<1.5$. The calculation of the antibody was according to the kit ((sample OD/cutoff serum mean $\mathrm{OD}) \times 10$ ). The interpretation of the test results was as follows: the serum sample $<9$ was negative, 9 - 11 was equivo$\mathrm{cal}$, and $>11$ was positive. This kit could identify individuals with previous infection and current infection.

\subsection{Statistical Analysis}

The statistical analysis of the data was done using SPSS version 19.0 software. The researchers calculated the ratios, percentages, and the chi-square to describe and analyze the data. The significance level was set at below 0.05 .

\section{Results}

In this study, a total of 184 samples from rural pregnant women with livestock contact were examined from December 2016 to June 2017 for the seroprevalence of $C$. burnetii. The minimum age of the subjects was 17 and the maximum age was 50 . The mean gestational age was 8.9 \pm 15.4 weeks. Of the total subjects, 126 (68.5\%) were under 30 years of age and 58 (31.5\%) were above 30. Moreover, 39 (21.2\%) women had a history of abortion. The majority of the women in the study $(\mathrm{n}=110 ; 59.8 \%)$ were in the first trimester of gestation, 65 (35.3\%) in the second trimester, and $9(4.9 \%)$ in the third trimester of pregnancy. The highest number of cases was reported to clinical health centers in the month of May $(\mathrm{n}=53 ; 28.8 \%)$ and the lowest in the month of June $(n=8 ; 4.3 \%)$. There were 58 (31.5\%) samples from the eastern villages of Khorramabad city, 30 (16.3\%) samples from western villages, 44 (23.9\%) samples from northern villages, and 52 (28.3\%) samples from southern villages. Table 1 shows the results of the serologic test of C. burnetii in rural pregnant women based on age and age of gestation. The positivity rate was $48.4 \%$ among under 30-year-old women and 48.3\% among upper 30-year-old women. This indicated no statistically significant difference $(\mathrm{P}$ value $=0.859)$. The positivity rate of $C$. burnetii was $46.6 \%$ in the first trimester of pregnancy, $50.8 \%$ in the second trimester, and $55.6 \%$ in the third trimester. This finding showed no statistically significant difference ( $P$ value $=0.451$ ). The number of positive samples of women with the experience of abortion is reported in Table 1. Among the group that had a history of abortion, 19 (48.7\%) samples were positive, which showed no statistically significant relationship $(\mathrm{P}$ value $=0.82)$. The number of positive samples according to the sampling month is presented in Table 2. The majority of the positive samples were from women who tested in January (83.3\%) and February (61.9\%). The least positive samples were from women who tested in June (12.5\%); this difference was statistically significant ( $P$ value $=0.001$ ). The differences between the locations of villages are shown in Table 2 . The results suggest that more positive samples were observed in women living in the eastern villages of Khorramabad city (compared to other villages); however, the result was not statistically significantly different $(P$ value $=0.535)$.

\section{Discussion}

$\mathrm{Q}$ fever has emerged as a newly emerging zoonosis in many countries, including Iran (10). Although $Q$ fever is endemic in Iran, little research has been carried out in this respect (11). This is the first study that investigated rural pregnant women in Iran. In this study, the seroprevalence of C. burnetii phase II was found positive at a rate of $48.4 \%$ in pregnant women with a history of contact with livestock. The high seroprevalence in this study might be due to the studied population (rural pregnant women) who had a long history of contact with livestock. The results of this study indicated that although ELISA is a sensitive method, it is better to use the IFA method as reference (8). However, the most commonly used method for the diagnosis of $\mathrm{Q}$ fever serology is ELISA in Iran (11). Some of the studies conducted inside and outside the country are as follows.

In 2011, Metanat et al. (12) designed a study in Zahedan in which 105 febrile patients with a probable diagnosis of brucellosis were investigated using the IFA technique for detecting $C$. burnetii antibodies in sera. The results showed that $35.2 \%$ of the patients had positive sera on the acute form of the fever and most people lived in rural areas. In this study, 52 males and 53 females were examined; most of the positive cases were women and the majority of men who were declared positive were livestock farmers. In a study, Khalili et al. (13) studied the prevalence and risk factors of $\mathrm{Q}$ fever among veterinary students in Kerman using the ELISA method. Among 121 sera samples, 42 (34.7\%) were 


\begin{tabular}{|c|c|c|c|c|c|}
\hline Factors & Positive & Negative & Suspect & Total & P Value \\
\hline Age & & & & & 0.859 \\
\hline$<30$ & $61(48.4)$ & $53(42.1)$ & $12(9.5)$ & $126(68.5)$ & \\
\hline$>30$ & $28(48.3)$ & $23(39.7)$ & $7(12.1)$ & $58(31.5)$ & \\
\hline $\begin{array}{l}\text { Age of gestation according } \\
\text { (week) }\end{array}$ & & & & & 0.451 \\
\hline 1-14 & $51(46.6)$ & $33(50.8)$ & $5(55.6)$ & $110(59.8)$ & \\
\hline $15-28$ & $33(50.8)$ & $28(43.1)$ & $4(6.2)$ & $65(35.3)$ & \\
\hline $29-40$ & $5(55.6)$ & $2(22.2)$ & $2(22.2)$ & $9(4.9)$ & \\
\hline History of abortion & & & & & 0.82 \\
\hline Yes & $19(48.7)$ & $15(38.5)$ & $5(12.8)$ & $39(21.2)$ & \\
\hline No & $70(48.3)$ & $61(42.1)$ & $14(9.7)$ & $145(78.8)$ & \\
\hline Variable & Positive & Negative & Suspect & Total & P Value \\
\hline Months type & & & & & 0.001 \\
\hline January & $25(83.3)$ & $4(13.3)$ & $1(3.3)$ & $30(16.3)$ & \\
\hline February & $26(61.9)$ & $11(26.2)$ & $5(11.9)$ & $42(22.8)$ & \\
\hline March & $11(39.3)$ & $12(42.9)$ & $5(17.9)$ & $28(15.2)$ & \\
\hline April & $6(26.1)$ & $14(60.9)$ & $3(13)$ & $23(12.5)$ & \\
\hline May & $20(37.7)$ & $28(52.8)$ & $5(9.4)$ & $53(28.8)$ & \\
\hline June & $1(12.5)$ & $7(87.5)$ & $0(0)$ & $8(4.3)$ & \\
\hline Location of villages & & & & & 0.535 \\
\hline North & $17(38.6)$ & $19(43.2)$ & $8(18.2)$ & $44(23.9)$ & \\
\hline South & $28(53.8)$ & $20(38.5)$ & $4(7.7)$ & $52(28.3)$ & \\
\hline East & $29(50)$ & $25(43.1)$ & $4(6.9)$ & $58(31.5)$ & \\
\hline West & $15(50)$ & $12(40)$ & $3(10)$ & $30(16.3)$ & \\
\hline
\end{tabular}

positive. The results of this study, based on the number of samples, showed a relatively high prevalence among veterinary students. In a 2014 study, Khayyat Khameneie et al. (9) conducted the first serological study of C. burnetii among pregnant women in Ahvaz and Parsabad on 400 samples (200 from Ahvaz and 200 from Parsabad) in which 44 samples (22\%) were positive in Ahvaz and 148 samples with pregnancy condition and 56 samples (37.83\%) were positive in Parsabad. The seroprevalence of $Q$ fever in pregnant women was higher in Parsabad than in Ahvaz, which may be due to the livestock and mountainous nature of Parsabad. Another point is that most people in Parsabad referring to laboratories were from rural areas. Other studies on women in Iran were conducted in various areas including Bardsir (20\%), Tabriz (13.8\%), South Khorasan (54.4\%), Kurdistan (38\%), and Kerman (68\%) (14-18). McCaughey et al. (19) studied Q fever seroprevalence from 1986 to 1987 in Northern Ireland by the ELISA method. They found that among 2,394 samples, the prevalence rate of $C$. burnetii was 12.8\%. Among 1,185 males, 170 (14.3\%) samples were positive and among 1,209 females, 136 (11.2\%) samples were positive. It was found that the prevalence of the disease was more in males than in females. The differences observed in this study were based on the total sample, the sampling time, and the studied population. In a study between 2007 and 2009, van der Hoek et al. (20) in the Netherlands examined the prevalence of $C$. burnetii among rural pregnant women by the IFA method. Of the 2004 pregnant women samples, 181(9\%) were positive. Considering the total number of people participating in this study and the total number of positive cases, it can be concluded that there is a low prevalence of $\mathrm{Q}$ fever in this area. A study by Baud et al. (21) in London in 2008 for the prevalence of C. burnetii in women by the IFA method showed that of the 438 serum 
samples tested, 20 (4.6\%) samples were positive. According to these results, there were low incidence rates compared to our study. In 2014, Fenga et al. (22) in Italy studied the seroprevalence in people exposed to livestock by the ELISA method. Of the 140 samples, 88 (62.9\%) were positive. According to the total number of samples in this study, a high prevalence of positive $\mathrm{Q}$ fever cases was reported, which can be compared with our study in terms of results and contact with livestock. In a study in Turkey in 2012, Gunal et al. (7) investigated the relationship between $\mathrm{Q}$ fever and abortion using the IFA method. Of 100 serum samples from women, 14 positive cases were reported.

The prevalence of $Q$ fever varies from country to country, as follows: China (35.6\%), Japan (2\%), France (2.6\%), and Bulgaria (7.7\%) (23-26). There is a considerable difference between recent studies and the conducted studies in Iran, possibly due to the conditions of studies, the time of sampling, the number of populations selected, and the number of samples selected for studies. One of the most important points, according to previous studies, is the presence of many $\mathrm{Q}$ fever cases in the livestock population in Khorramabad city $(27,28)$, which could be due to the differences between the study and other studies. In studies conducted around the world, there is a key difference with recent studies; a controversy is the use of the IFA diagnostic technique and studying a wide range of people, which lead to the large collection of samples and the sampling time, which usually take more than a year or even several years. The climate conditions for transmission, direct and indirect contact with livestock and animals' products, and management practices in dealing with disease and treatment are important factors influencing the results of studies. However, there is not enough awareness about the disease as demonstrated by recent records in Iran. The disease is largely ignored due to the lack of distinction between clinical cases and illnesses such as malt fever and influenza, which indicates that medical communities are not aware of the disease. Therefore, healthcare centers need to be provided with inclusive and continuous training to be aware of the dangers of the disease.

\subsection{Conclusions}

The present study showed that $Q$ fever was observed in Iranian rural pregnant women who had a history of contact with livestock. Therefore, since $\mathrm{Q}$ fever is a risk factor for pregnant women, medical policymakers and the Ministry of Health and Medical Education should raise individuals' awareness of the dangerous effects of the disease, especially for those who have frequent contact with livestock. To prevent and control the spread of infection between humans and livestock, it is essential to timely vaccinate livestock against the disease. Moreover, milk pasteur- ization is a helpful way to prevent human contamination and related diseases.

\section{Acknowledgments}

The present study is based on a dissertation. The project executives acknowledge and appreciate the support and cooperation of the health centers of Khorramabad city and Lorestan University. The researchers also thank Fariba Hadipanah who helped us in the process of sampling.

\section{Footnotes}

Authors' Contribution: eiman Azizyar Ghobadi did drafting of the manuscript, analysis and interpretation of data. Amin Jaydari did study concept and design, drafting of the manuscript, analysis and interpretation of data, study supervision. Soheila Akbari did technical and material support. Khaterh Anbari did statistical analysis.

Conflict of Interests: It is not declared by authors.

Ethical Approval: The Ethics Committee of Lorestan University of Medical Sciences approved the study (http://vcr.lums.ac.ir IR.LUMS.REC 2016.175).

Funding/Support: The study has been funded by Lorestan University granted the study.

Informed Consent: The participants provided written informed consent for the publication.

\section{References}

1. Maurin M, Raoult D. Q fever. Clin Microbiol Rev. 1999;12(4):518-53. [PubMed: 10515901]. [PubMed Central: PMC88923].

2. van Schaik EJ, Chen C, Mertens K, Weber MM, Samuel JE. Molecular pathogenesis of the obligate intracellular bacterium Coxiella burnetii. Nat Rev Microbiol. 2013;11(8):561-73. doi: 10.1038/nrmicro3049. [PubMed: 23797173]. [PubMed Central: PMC4134018].

3. Marrie TJ. Epidemiology of Q fever. UK: CRC, Boca Raton, FL;1995. p. 4970.

4. Psaroulaki A, Hadjichristodoulou C, Loukaides F, Soteriades E, Konstantinidis A, Papastergiou P, et al. Epidemiological study of Q fever in humans, ruminant animals, and ticks in Cyprus using a geographical information system. Eur J Clin Microbiol Infect Dis. 2006;25(9):576-86. doi: 10.1007/s10096-006-0170-7. [PubMed:16915398].

5. Rey D, Obadia Y, Tissot-Dupont H, Raoult D. Seroprevalence of antibodies to Coxiella burnetti among pregnant women in South Eastern France. Eur J Obstet Gynecol Reprod Biol. 2000;93(2):151-6. doi: 10.1016/s0301-2115(00)00276-1. [PubMed: 11074136].

6. Carcopino X, Raoult D, Bretelle F, Boubli L, Stein A. Managing Q fever during pregnancy: The benefits of long-term cotrimoxazole therapy. Clin Infect Dis. 2007;45(5):548-55. doi: 10.1086/520661. [PubMed: 17682987].

7. Gunal O, Demirtürk F, Barut S, Kilic S, Erkorkmaz U, Tekin FY, et al. A preliminary report of relationship between abortion and Q fever in central Black Sea region Turkish woman. Cumhuriyet Med J. 2014;36(3):337. doi: 10.7197/cmj.v36i3.5000013124. 
8. Marrie TJ, Raoult D. Coxiella burnetii (Q Fever). In: Mandell GL, Bennett JE, Dolin R, editors. Principles and practice of clinical infectious diseases. 7th ed. Philadelphia, PA. USA: Churchill Livingstone; 2010. p 2511-9. doi: 10.1016/b978-0-443-06839-3.00189-2.

9. Khayyat Khameneie M, Asadi J, Khalili M, Abiri Z. The first serological study of Coxiella burnetii among pregnant women in Iran. Iran J Public Health. 2016;45(4):523-30. [PubMed: 27252922]. [PubMed Central: PMC4888180].

10. Pourmahdi Borujeni M, Gharibi D, Gouranejad S, Zamiri S. Seroprevalence of coxiellosis in Ahvaz sheep. Iran Vet J. 2013;9(1):11-8.

11. Mohabbati Mobarez A, Bagheri Amiri F, Esmaeili S. Seroprevalence of $\mathrm{Q}$ fever among human and animal in Iran; a systematic review and meta-analysis. PLoS Negl Trop Dis. 2017;11(4). e0005521. doi: 10.1371/journal.pntd.0005521. [PubMed: 28394889]. [PubMed Central: PMC5398711].

12. Metanat M, Sepehri Rad N, Alavi-Naini R, Shahreki S, Sharifi-Mood B, Akhavan A, et al. Acute Q fever among febrile patients in Zahedan, southeastern Iran. Turk J Med Sci. 2014;44(1):99-103. doi: 10.3906/sag 1209-102. [PubMed: 25558567].

13. Khalili M, Qorbani A, Sharifi H, Golchin M. Prevalence and risk factor of $\mathrm{Q}$ fever among veterinary students in Iran. Trop Biomed. 2015;32(4):704-9.

14. Naderipour Z, Golchin M, Khalili M. Design of an ELISA kit for detection human acute Q fever. Iran J Med Microbiol. 2014;8(2):28-34.

15. Esmaeili S, Golzar F, Ayubi E, Naghili B, Mostafavi E. Acute Q fever in febrile patients in northwestern of Iran. PLoS Negl Trop Dis. 2017;11(4) e0005535. doi: 10.1371/journal.pntd.0005535. [PubMed: 28394892]. [PubMed Central: PMC5398717].

16. Khalili M, Aflatoonian MR, Rahanjam M, Golchin M, Sharifi H. Frequency of seropositivity for anti-Coxiella burnetii (phase ii) among veterinary staff in Southern Khorasan, Iran, in 2014.J Kerman Univ Med Sci. 2016;23:164-73.

17. Esmaeili S, Pourhossein B, Gouya MM, Amiri FB, Mostafavi E Seroepidemiological survey of $\mathrm{Q}$ fever and brucellosis in Kurdistan Province, western Iran. Vector Borne Zoonotic Dis. 2014;14(1):41-5 doi: 10.1089/vbz.2013.1379. [PubMed: 24359427]. [PubMed Central: PMC3880925].

18. Khalili M, Mosavi M, Diali HG, Mirza HN. Serologic survey for Coxiella burnetii phase II antibodies among slaughterhouse workers in Kerman, southeast of Iran. Asian Pac J Trop Biomed. 2014;4(Suppl 1):S20912. doi: 10.12980/APJTB.4.2014C1268. [PubMed: 25183082]. [PubMed Central: PMC4025290].
19. McCaughey C, McKenna J, McKenna C, Coyle PV, O'Neill HJ, Wyatt DE, et al. Human seroprevalence to Coxiella burnetii (Q fever) in Northern Ireland. Zoonoses Public Health. 2008;55(4):189-94. doi: 10.1111/j.18632378.2008.01109.x. [PubMed:18387140].

20. van der Hoek W, Meekelenkamp JC, Dijkstra F, Notermans DW, Bom B, Vellema P, et al. Proximity to goat farms and Coxiella burnetii seroprevalence among pregnant women. Emerg Infect Dis. 2011;17(12):2360-3. doi: 10.3201/eid1712.110738. [PubMed: 22172140]. [PubMed Central: PMC3311170].

21. Baud D, Peter O, Langel C, Regan L, Greub G. Seroprevalence of Coxiella burnetii and Brucella abortus among pregnant women. Clin Microbiol Infect. 2009;15(5):499-501. doi:10.1111/j.1469-0691.2009.02779.x. [PubMed: 19489927].

22. Fenga C, Gangemi S, De Luca A, Calimeri S, Lo Giudice D, Pugliese $\mathrm{M}$, et al. Seroprevalence and occupational risk survey for Coxiella burnetii among exposed workers in Sicily, Southern Italy. Int J Occup Med Environ Health.2015;28(5):901-7. doi:10.13075/ijomeh.1896.00448. [PubMed: 26224501].

23. Sun WW, Cong W, Li MH, Wang CF, Shan XF, Qian AD. Coxiella burnetii seroprevalence and risk factors in cattle farmers and farm residents in three northeastern provinces and inner mongolia autonomous region, China. Biomed Res Int. 2016;2016:7059196. doi: 10.1155/2016/7059196. [PubMed: 26966688]. [PubMed Central: PMC4757716].

24. Numazaki K, Ueno H, Yokoo K, Muramatsu Y, Chiba S, Morita C. Detection of serum antibodies to Bartonella henselae and Coxiella burnetii from Japanese children and pregnant women. Microbes Infect. 2000;2(12):1431-4. doi: 10.1016/s1286-4579(00)01297-1. [PubMed: 11099929].

25. Langley JM, Marrie TJ, Leblanc JC, Almudevar A, Resch L, Raoult D. Coxiella burnetii seropositivity in parturient women is associated with adverse pregnancy outcomes. Am J Obstet Gynecol. 2003;189(1):228-32. doi: 10.1067/mob.2003.448. [PubMed:12861167].

26. Panaiotov S, Ciccozzi M, Brankova N, Levterova V, Mitova-Tiholova M, Amicosante M, et al. An outbreak of Q fever in Bulgaria. Ann Ist Super Sanita. 2009;45(1):83-6. [PubMed: 19567983].

27. Lorestani S, Jaydari A, Maleki S, Khademi P. Genomic detection of Coxiella burnetii in sheep milk samples by Nested-PCR method in Khorramabad, Iran. Food Sci Technol. 2015;13(56):165-71.

28. Khademi P, Mahzounieh MR, Kahrizsangi AE, Shdravan E. Genomic detection of Coxiella burnetii in goat milk samples in animal farms Khorramabad Township, Iran. Pajoohandeh J. 2014;19(3):162-8. 